increasing the maturation of miR-16 from its precursor pre/pri-miR-16. Raphe additionally responds to chronic fluoxetine treatment by releasing $S 100 \beta$, which in turn acts on the noradrenergic neurons of the locus coeruleus. By lowering miR-16 levels, S100 $\beta$ unlocks the expression of serotonergic functions in this noradrenergic brain area. Our pharmacological and behavioral data thus posit miR-16 as a central effector that regulates SERT expression and mediates the adaptive response of serotonergic and noradrenergic neurons to fluoxetine treatment.

\section{References and Notes}

1. G. E. Torres, R. R. Gainetdinov, M. G. Caron, Nat. Rev. Neurosci. 4, 13 (2003).

2. E. C. Azmitia, Int. Rev. Neurobiol. 77, 31 (2007).

3. O. Berton, E. J. Nestler, Nat. Rev. Neurosci. 7, 137 (2006).
4. Y. Qian, H. E. Melikian, D. B. Rye, A. I. Levey, R. D. Blakely, J. Neurosci. 15, 1261 (1995).

5. D. L. Murphy et al., Neuropharmacology 55, 932 (2008).

6. S. Benmansour, W. A. Owens, M. Cecchi, D. A. Morilak, A. Frazer, J. Neurosci. 22, 6766 (2002).

7. D. P. Bartel, Cell 136, 215 (2009).

8. C. M. Croce, Nat. Rev. Genet. 10, 704 (2009).

9. K. S. Kosik, Nat. Rev. Neurosci. 7, 911 (2006).

10. V. K. Gangaraju, H. Lin, Nat. Rev. Mol. Cell Biol. 10, 116 (2009).

11. S. Mouillet-Richard et al., J. Biol. Chem. 275, 9186 (2000).

12. J. M. Launay, B. Schneider, S. Loric, M. Da Prada, O. Kellermann, FASEB J. 20, 1843 (2006).

13. A. Frazer, J. Clin. Psychopharmacol. 17 (suppl. 1), 2S (1997).

14. E. J. Nestler, M. Alreja, G. K. Aghajanian, Biol. Psychiatry 46, 1131 (1999).

15. G. Martello et al., Nature 449, 183 (2007).

16. M. A. Kim, H. S. Lee, B. Y. Lee, B. D. Waterhouse, Brain Res. 1026, 56 (2004).

17. N. Shanmugam, M. A. Reddy, R. Natarajan, J. Biol. Chem. 283, 36221 (2008).
18. R. Manev, T. Uz, H. Manev, Eur. J. Pharmacol. 420, R1 (2001).

19. A. Surget et al., Neuropsychopharmacology 34, 1363 (2009).

20. P. Willner, Psychopharmacology (Berlin) 134, 319 (1997).

21. Materials and methods and supporting data are available on Science Online.

22. We thank P. Weil-Malherbe, V. Mutel, F. d'Agostini, G. Zürcher, E. Borroni, J. L. Moreau, F. Jenck, M. Bühler, and N. Pieron for skillful methodological assistance, and S. Blanquet, M. Briley, and L. Aggerbeck for critical reading of the manuscript. O.K. is a professor at Paris XI University. This work was funded by CNRS, ANR, and INSERM.

\section{Supporting Online Material}

www.sciencemag.org/cgi/content/full/329/5998/1537/DC1

Materials and Methods

Figs. S1 to S10

References

14 June 2010; accepted 4 August 2010

$10.1126 /$ science. 1193692

\title{
Relating Introspective Accuracy to Individual Differences in Brain Structure
}

\section{Stephen M. Fleming, ${ }^{1 *} \dagger$ Rimona S. Weil, ${ }^{1,2 *}$ Zoltan Nagy, ${ }^{1}$ Raymond ]. Dolan, ${ }^{1}$ Geraint Rees ${ }^{1,2}$}

The ability to introspect about self-performance is key to human subjective experience, but the neuroanatomical basis of this ability is unknown. Such accurate introspection requires discriminating correct decisions from incorrect ones, a capacity that varies substantially across individuals. We dissociated variation in introspective ability from objective performance in a simple perceptualdecision task, allowing us to determine whether this interindividual variability was associated with a distinct neural basis. We show that introspective ability is correlated with gray matter volume in the anterior prefrontal cortex, a region that shows marked evolutionary development in humans. Moreover, interindividual variation in introspective ability is also correlated with white-matter microstructure connected with this area of the prefrontal cortex. Our findings point to a focal neuroanatomical substrate for introspective ability, a substrate distinct from that supporting primary perception.

$\mathrm{O}$ ur moment-to-moment judgments of the outside world are often subject to introspective interrogation. In this context, introspective or "metacognitive" sensitivity refers

${ }^{1}$ Wellcome Trust Centre for Neuroimaging, University College London, 12 Queen Square, London WC1N 3BG, UK. ' Institute of Cognitive Neuroscience, University College London, 17 Queen Square, London WC1N 3AR, UK.

*These authors contributed equally to this work. †To whom correspondence should be addressed. E-mail: s.fleming@fil.ion.ucl.ac.uk to the ability to discriminate correct from incorrect perceptual decisions (1), and its accuracy is essential for the appropriate guidance of decision-making and action $(2,3)$. For example, low confidence that a recent decision was correct may prompt us to reexamine the evidence or seek a second opinion. Recently, behavioral studies have begun to quantify metacognitive accuracy following simple perceptual decisions and to isolate variations in this ability: A decision may be made poorly, yet an individual may believe that his or her performance was good, or vice versa (4-8). Whereas previous work has investigated how confidence in perceptual decisions varies from trial to trial $(9,10)$, little is known about the biological basis of metacognitive ability, defined here as how well an individual's confidence ratings discriminate correct from incorrect decisions over time. We hypothesized that individual differences in metacognitive ability would be reflected in the anatomy of brain regions responsible for this function, in line with similar associations between brain anatomy and performance in other cognitive domains (11-15).

We objectively quantified variability in metacognitive sensitivity between individuals and then related these interindividual differences to brain structure measured with magnetic resonance imaging (MRI). This approach was motivated by observations that individual differences in a range of skills - such as language (11), decision-making (12), and memory (13) - are consistently associated with variation in healthy brain anatomy. Our experimental design dissociated a quantitative measure of metacognitive accuracy, $A_{\text {roc }}$ (which is specific to an individual), from both objective task performance and subjective confidence (which both vary on a trial-by-trial basis). Earlier patient studies describe candidate brain regions in which damage is associated with poor introspective ability: in particular, a prefrontalparietal network (16-18). Theories of prefrontal

Fig. 1. Behavioral task. Participants completed a two-alternative forced-choice task that required two judgments per trial: a perceptual response followed by an estimate of relative confidence in their decision. The perceptual response indicated whether the first or second temporal interval contained the higher-contrast (popout) Gabor patch (highlighted here with a dashed circle that was not present in the actual display), which could appear at any one of six locations around a central fixation point. Pop-out Gabor contrast was continually adjusted with the use of a staircase procedure to maintain $\sim 71 \%$ performance. Confidence ratings were

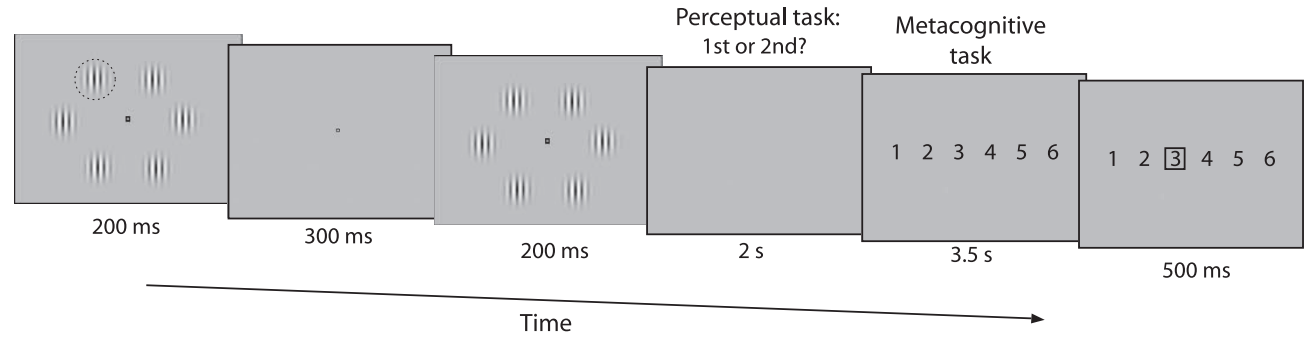

made using a one-to-six scale, with participants encouraged to use the whole scale from one $=$ low relative confidence to six $=$ high relative confidence. The black square in the rightmost panel indicates the choice made in the metacognitive task. 
function have emphasized a role for anterior (rostrolateral) prefrontal cortex (PFC) in carrying out second-order operations on internally generated information $(19,20)$, a process necessary for metacognition. We hypothesized that the local structure of these regions (both gray-matter volume and white-matter integrity) might reflect an individual's metacognitive ability.

We studied 32 healthy human participants while they made a series of visual judgements (21). The difficulty of the visual judgement was varied on a per-participant basis to keep performance at a constant level (71\%), near sensory threshold. In addition to asking participants to make these objective perceptual judgements, we also asked them to provide ratings of confidence in their decisions after each trial (Fig. 1). We then used these ratings to determine metacognitive ability at an individual level through the construction of type II receiver operating characteristic (ROC) curves (Fig. 2A) (21-23). The ROC model provided an excellent fit to our data across participants (mean explained variance $R^{2}=0.97 \pm$ 0.023 ). The area between the major diagonal and an individual's ROC curve is a measure of the ability to link confidence to perceptual performance $\left(A_{\text {roc }}\right)$. We found considerable variation across individuals in metacognitive ability $\left(A_{\mathrm{roc}}=\right.$ 0.55 to 0.75 ), despite underlying task performance being held constant (proportion correct: 70 to $74 \%$ ); furthermore, these measures were uncorrelated (Pearson's correlation coefficient $r=$ $-0.21, P=0.24)$. To establish whether this variability was stable, we split data from each participant into two halves and computed the test-retest reliability of the two sets. This analysis revealed intraparticipant consistency in $A_{\text {roc }}(r=$ $0.69, P=0.00001$ ) (fig. S2).

Having quantified interindividual variability in introspection, we then asked whether this variability in introspective judgements was predicted by variability in brain structure using two distinct measures: gray-matter volume measured from T1weighted anatomical images and the fractional anisotropy (FA) of white matter measured from diffusion tensor images. Our analysis examined the possible relation between brain structure and four different measures: the metacognitive ability $\left(A_{\text {roc }}\right)$ of our participants, objective performance on the perceptual task (sensitivity, $d^{\prime}$, and criterion, $c$ ), and the tendency to use high or low confidence responses on individual trials $\left(B_{\text {roc }}\right)$ [see supporting online material (SOM) methods section for details]. Having removed the potentially confounding factors (24) of overall brain size and gender (as regressors of no interest), we found that an individual's metacognitive ability $\left(A_{\text {roc }}\right)$ was significantly correlated with gray-matter volume in the right anterior PFC (Fig. 3A) [Brodmann area (BA) 10; peak voxel coordinates: $[24,65,18]$; $t_{\max }=$ 4.8; $P<0.05$, corrected for multiple comparisons]. Furthermore, gray-matter volume in this region did not correlate with task performance, as indexed by $d^{\prime}$ (Fig. 3B) $(r=0.15, P=0.42)$, or overall confidence $\left(B_{\mathrm{roc}}\right)(r=-0.023, P=0.90)$.

A

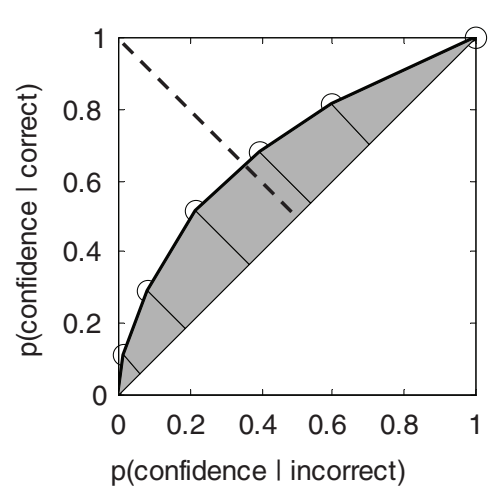

B

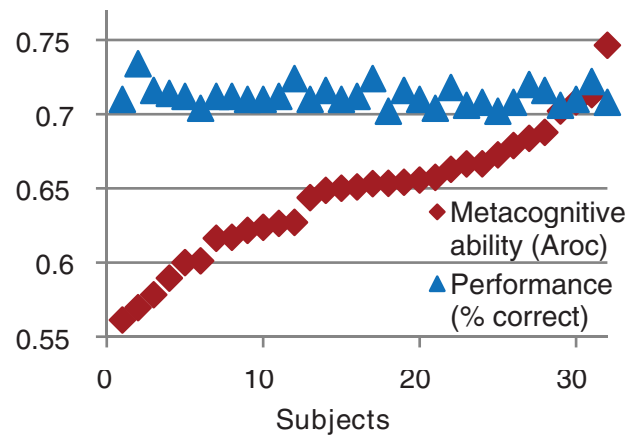

Fig. 2. ROC calculation and behavioral performance. (A) Participants' confidence ratings were used to construct a type II ROC function that quantifies the ability to discriminate between correct and incorrect responses cumulated across levels of confidence. $A_{\text {roc }}$ was calculated as the shaded area between the ROC curve and the major diagonal (21). Mutually perpendicular dotted and solid lines represent the minor and major diagonals, respectively. (B) Plot of the relation between task performance (percentage correct) and $A_{\text {roc }}$ with participants ordered by increasing $A_{\text {roc }}$ value.

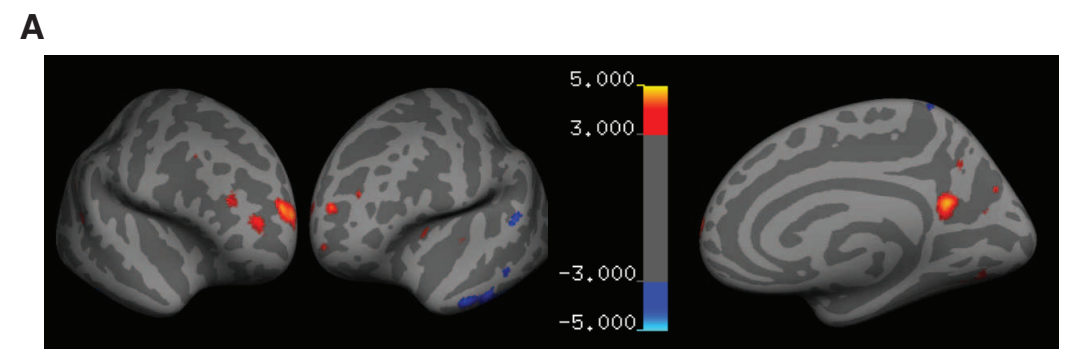

B

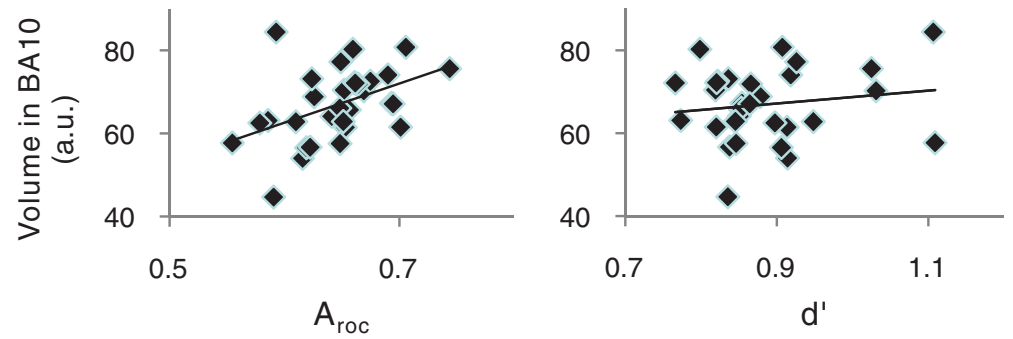

Fig. 3. Gray-matter volume correlated with introspective ability. (A) Projection of statistical (T) maps for positive (hot color map: red, orange, yellow) and negative (cool color map: blue) correlations with $A_{\text {roc }}$ onto an inflated cortical surface (T1-weighted template, thresholded at $T>3$ for display purposes). Significant clusters ( $P<0.05$, corrected for multiple comparisons) where metacognitive ability correlated with gray-matter volume (see SOM methods) were found in right anterior PFC (BA 10; positive correlation) and the left inferior temporal gyrus (negative correlation), accompanied by contralateral homologous clusters at $P<0.001$, uncorrected. (B) Plot of gray-matter volume in the right BA 10 cluster against both $A_{\text {roc }}$ and $d^{\prime}$ (see SOM methods for full details), indicating that the correlation with metacognitive ability was independent of task performance. a.u., arbitrary units.

Gray-matter volume in a homologous region in the left anterior PFC was also correlated with $A_{\text {roc }}$ but did not survive correction for multiple comparisons across the brain volume. Details of this and other clusters that did not survive a whole-brain correction are listed in table S2. Thus, variability in introspective judgements of performance on a simple visual-detection task was predicted by variability in the anatomical structure of the anterior PFC (BA 10), independently of both objective performance and level of confidence. Finally, whereas our primary question addressed positive dependence of gray matter on $A_{\text {roc }}$, we also found that the left inferior tem- poral gyrus showed a negative correlation with metacognitive sensitivity (Fig. 3A) (coordinates: $[-56,-30,-26] ; t_{\max }=4.66 ; P<0.05$, corrected for multiple comparisons), accompanied by a similar region on the right that did not survive correction for multiple comparisons (see table S2 for full details and coordinates).

After we established that gray-matter volume was predictive of $A_{\text {roc }}$, we next analyzed whitematter microstructure. If the structure of the anterior PFC is functionally related to metacognitive performance, we hypothesized that white-matter tracts connected with this region would also show a similar microstructural correlation with 
Fig. 4. White-matter microstructure correlated with introspective ability. (A) Statistical (T) map of voxelwise correlations between FA and $A_{\text {roc }}$ thresholded at $T>3$ for display purposes and overlaid on sagittal (left) and axial (right) slices of the average FA image across participants, at the $x$ and $z$ coordinates indicated. A region within the genu of the anterior corpus callosum showed a correlation between FA and metacognitive ability that was statistically significant after correcting for multiple comparisons $(P<$ 0.05). (B) Plot of FA in the anterior corpus callosum cluster against both $A_{\text {roc }}$ and $d^{\prime}$, indicating that the correlation with metacognitive ability was independent of task performance.

expression of this behavioral trait. In a wholebrain analysis of white-matter microstructure (21), we found that FA (a measure of whitematter integrity) in the genu of the corpus callosum was positively dependent on $A_{\text {roc }}$ (Fig. 4) $(P<0.05$, corrected for multiple comparisons). This specific subdivision of the corpus callosum contains white-matter fibers connected with the anterior and orbital PFCs in humans (25), consistent with metacognitive ability being dependent not only on anterior prefrontal gray matter but also on reciprocal projections to and from this area. Neither objective performance (stimulus contrast or $\left.d^{\prime}\right)$ nor overall confidence $\left(B_{\mathrm{roc}}\right)$ correlated with gray-matter volume or white-matter FA elsewhere in the brain $(P>0.05$, corrected for multiple comparisons; see tables S2 and S3 for uncorrected correlations). We note that an absence of structural correlations with these parameters may have been due to our design deliberately minimizing variability in both $d^{\prime}$ and $B_{\text {roc }}$ to isolate the neural correlates of introspective ability $\left(A_{\mathrm{roc}}\right)$.

One concern is that the structural covariation that we observed may have been potentially confounded by differences in perceptual ability. Good perceptual ability may be reflected in the staircase procedure converging on consistently low values for stimulus contrast for a given individual. Therefore, we carried out control analyses (table S4) (21) to rule out this alternative explanation. These results demonstrated significant correlations of gray matter and FA with $A_{\text {roc }}$ in the anterior PFC when controlling for changes in task parameters and an absence of correlations with task parameters themselves. Thus, the structurebehavior correlations we observed here are unlikely to be due to low-level differences in performance, but instead relate to underlying differences in individual metacognitive ability.

How might these regions contribute to metacognition? Anterior subdivisions of the PFC have been implicated in high-level control of cognition $(19,20,26,27)$ and are well placed to integrate supramodal perceptual information with decision output (28), a process thought to be key for metacognitive sensitivity (1). Dorsolateral prefrontal activity increases under conditions in which subjective reports match objective perceptual performance (29), suggesting a computational role in linking performance to confidence. Consistent with prefrontal gray-matter volume playing a causal role in metacognition, patients with lesions to the anterior PFC show deficits in subjective reports as compared with controls, after factoring out differences in objective performance (16). Furthermore, impairing dorsolateral PFC function with theta-burst transcranial magnetic stimulation compromises the metacognitive sensitivity of subjective reports of awareness but leaves underlying task performance intact (30). Together with the present work, these findings suggest a central role for anterior and dorsolateral PFC in metacognitive sensitivity. Our present findings may reflect innate differences in anatomy or, alternatively, may reflect the effects of experience and learning, as has been found in the sensorimotor domain $(14,15)$. This raises the tantalizing possibility of being able to "train" metacognitive ability by harnessing underlying neural plasticity in the regions that we identify here (31).

Our main finding is a delineation of a noticeably focal anatomical substrate that predicts interindividual variability in metacognitive ability. As with any correlational method, we cannot establish whether the covariation we observed between brain structure and metacognition reflects a causal relation. However, given a wealth of evidence for changes in gray-matter volume within and between individuals associated with a range of skills, we propose that underlying differences in metacognitive ability are similarly dependent on large-scale brain anatomy. Our data provide an ini- tial window to the biological basis of the ability to link objective performance to subjective confidence. The demonstration that this ability may be dependent on local and phylogenetically recent prefrontal anatomy is consistent with a conjecture that metacognitive function has been selected for during evolution (32), facilitating computations that allow us to introspect about self-performance.

\section{References and Notes}

1. A. Cleeremans, B. Timmermans, A. Pasquali, Neural Networks 20, 1032 (2007).

2. ]. Metcalfe, Metacognition: Knowing About Knowing (MIT Press, Cambridge, MA, 1996).

3. H. C. Lau, Prog. Brain Res. 168, 35 (2007).

4. D. A. Washburn, J. D. Smith, L. A. Taglialatela, J. Gen. Psychol. 132, 446 (2005).

5. C. Kunimoto, J. Miller, H. Pashler, Conscious. Cogn. 10, 294 (2001).

6. R. Szczepanowski, L. Pessoa, J. Vis. 7, 1 (2007)

7. M. Graziano, M. Sigman, A. Rustichini, PLOS ONE 4, e4909 (2009)

8. S. M. Fleming, R. J. Dolan, Conscious. Cogn. 19, 352 (2010).

9. R. Kiani, M. N. Shadlen, Science 324, 759 (2009).

10. A. Kepecs, N. Uchida, H. A. Zariwala, Z. F. Mainen, Nature 455, 227 (2008).

11. M. Carreiras et al., Nature 461, 983 (2009).

12. D. S. Tuch et al., Proc. Natl. Acad. Sci. U.S.A. 102, 12212 (2005).

13. L. Fuentemilla et al., J. Neurosci. 29, 8698 (2009).

14. J. Scholz, M. C. Klein, T. E. J. Behrens, H. Johansen-Berg, Nat. Neurosci. 12, 1370 (2009).

15. B. Draganski et al., Nature 427, 311 (2004).

16. A. Del Cul, S. Dehaene, P. Reyes, E. Bravo, A. Slachevsky, Brain 132, 2531 (2009)

17. A. P. Shimamura, Conscious. Cogn. 9, 313 (2000).

18. J. S. Simons, P. V. Peers, Y. S. Mazuz, M. E. Berryhill, I. R. Olson, Cereb. Cortex 20, 479 (2010).

19. P. C. Fletcher, R. N. A. Henson, Brain 124, 849 (2001).

20. K. Christoff, J. D. E. Gabrieli, Psychobiology 28, 168 (2000).

21. Materials, methods, discussion of ROC model fits, and details of control analyses are available as supporting material on Science Online.

22. S. J. Galvin, J. V. Podd, V. Drga, ]. Whitmore, Psychon Bull. Rev. 10, 843 (2003).

23. D. E. Kornbrot, Percept. Psychophys. 68, 393 (2006).

24. C. D. Smith, H. Chebrolu, D. R. Wekstein, F. A. Schmitt, W. R. Markesbery, Neurobiol. Aging 28, 1075 (2007).

25. H. J. Park et al., Hum. Brain Mapp. 29, 503 (2008)

26. N. D. Daw, J. P. O'Doherty, P. Dayan, B. Seymour, R. J. Dolan, Nature 441, 876 (2006).

27. P. W. Burgess, I. Dumontheil, S. J. Gilbert, Trends Cogn. Sci. 11, 290 (2007).

28. N. Ramnani, A. M. Owen, Nat. Rev. Neurosci. 5, 184 (2004).

29. H. C. Lau, R. E. Passingham, Proc. Natl. Acad. Sci. U.S.A. 103, 18763 (2006)

30. E. Rounis et al., Cognit. Neurosci. 1, 165 (2010).

31. E. B. Titchener, Lectures on the Experimental Psychology of the Thought-Processes (Macmillan, New York, 1909).

32. ]. Metcalfe, in Handbook of Metamemory and Memory, J. Dunlosky, R. A. Bjork, Eds. (Psychology Press, New York, 2008), pp. 27-46.

33. This research was funded by the Wellcome Trust (G.R., R.J.D., Z.N.), the University College London four-year Ph.D. program in neuroscience (S.M.F.) and the Medical Research Council (R.S.W.). We thank T. Sharot, G. Ridgway, and C. Frith for comments on earlier drafts of this manuscript.

\section{Supporting Online Material}

www.sciencemag.org/cgi/content/full/329/5998/1541/DC1

Materials and Methods

SOM Text

Figs. $\mathrm{S} 1$ to $\mathrm{S4}$

Tables S1 to S4

References

5 May 2010; accepted 23 July 2010

10.1126/science. 1191883 
Reports: "Relating introspective accuracy to individual differences in brain structure" by S. M. Fleming et al. (17 September 2010, p. 1541). The authors have detected a minor programming error in their original analysis of brain structure. Thus, spatial coordinates for a subset of results should be corrected by $\pm 2 \mathrm{~mm}$ (see the table below for the correct coordinates). No other aspects of their results or interpretations are affected.

\begin{tabular}{|c|c|c|c|}
\hline Contrast & Region & $\begin{array}{l}\text { Peak voxel } \\
\text { reported in paper } \\
\text { (MNI) }\end{array}$ & $\begin{array}{l}\text { Corrected peak } \\
\text { voxel (MNI) }\end{array}$ \\
\hline GM-positive $A_{\text {roc }}$ & Right BA10 & $24,65,18$ & $26,66,16$ \\
\hline GM-positive $A_{\mathrm{roc}}$ & Precuneus & $6,-57,18$ & $8,-57,19$ \\
\hline WM-positive $A_{\text {roc }}$ & $\begin{array}{l}\text { Anterior corpus } \\
\text { callosum }\end{array}$ & $2,26,-2$ & $2,27,-2$ \\
\hline
\end{tabular}

MNI, Montreal Neurological Institute; GM , grey matter volume; WM, white matter (fractional anisotropy); $A_{\text {roc }}$, a quantitative measure of metacognitive accuracy. 


\section{Relating Introspective Accuracy to Individual Differences in Brain Structure}

Stephen M. Fleming et al.

Science 329, 1541 (2010);

MIAAAS

DOI: $10.1126 /$ science. 1191883

This copy is for your personal, non-commercial use only.

If you wish to distribute this article to others, you can order high-quality copies for your colleagues, clients, or customers by clicking here.

Permission to republish or repurpose articles or portions of articles can be obtained by following the guidelines here.

The following resources related to this article are available online at www.sciencemag.org (this information is current as of February 20, 2015 ):

A correction has been published for this article at: http://www.sciencemag.org/content/336/6082/670.full.html

Updated information and services, including high-resolution figures, can be found in the online version of this article at:

http://www.sciencemag.org/content/329/5998/1541.full.html

Supporting Online Material can be found at:

http://www.sciencemag.org/content/suppl/2010/09/14/329.5998.1541.DC1.html

A list of selected additional articles on the Science Web sites related to this article can be found at:

http://www.sciencemag.org/content/329/5998/1541.full.html\#related

This article cites $\mathbf{2 8}$ articles, 8 of which can be accessed free:

http://www.sciencemag.org/content/329/5998/1541.full.html\#ref-list-1

This article has been cited by 45 articles hosted by HighWire Press; see:

http://www.sciencemag.org/content/329/5998/1541.full.html\#related-urls

This article appears in the following subject collections:

Neuroscience

http://www.sciencemag.org/cgi/collection/neuroscience 


\title{
Supporting Online Material for
}

\section{Relating Introspective Accuracy to Individual Differences in Brain Structure}

\author{
Stephen M. Fleming, * Rimona S. Weil, Zoltan Nagy, Raymond J. Dolan, Geraint Rees \\ *To whom correspondence should be addressed. E-mail: s.fleming@fil.ion.ucl.ac.uk
}

Published 17 September 2010, Science 329, 1541 (2010)

DOI: 10.1126/science.1191883

This PDF file includes:

Materials and Methods

SOM Text

Figs. S1 to S4

Tables S1 to S4

References 


\section{Materials and Methods}

\section{Participants}

32 participants (15 males; aged 19 - 37 years; mean age 26.4 years) gave written informed consent to take part in the experiment. The study was approved by the local Research Ethics Committee. One participant was excluded from further analysis of brain structure due to aberrant psychophysical task performance $\left(d^{\prime}>3 \mathrm{SD}\right.$ from the group mean).

\section{Stimuli}

The perceptual decision display comprised six Gabor gratings (circular patches of smoothly varying light and dark bars) arranged around a central fixation point (Fig 1). Each Gabor subtended 1.4 degrees of visual angle in diameter, and consisted of a luminance pattern modulated at a spatial frequency of 2.2 cycles per degree. Each "baseline" Gabor had a contrast of $20 \%$ of maximum, and appeared at a mean eccentricity of 6.9 degrees. The fixation point comprised a black square measuring 0.2 degrees across, luminance $0.10 \mathrm{~cd} / \mathrm{m}^{2}$, with a central white square 0.1 degrees across, luminance $13.64 \mathrm{~cd} / \mathrm{m}^{2}$. The background was a uniform gray screen of luminance 3.66 $\mathrm{cd} / \mathrm{m}^{2}$.

Baseline Gabors were displayed with a contrast of $20 \%$ (where $0 \%$ is no difference between the luminance of the grating bars and $100 \%$ is maximum difference, i.e. black to white). The pop-out Gabors were drawn from a stimulus set in which contrast varied from 23 to $80 \%$ in increments of $3 \%$. At the time of confidence ratings, the display consisted of a grey screen (luminance $3.66 \mathrm{~cd} / \mathrm{m}^{2}$ ) with the numbers 1 to 6 written left to right (luminance $13.64 \mathrm{~cd} / \mathrm{m}^{2}, 0.7$ degrees in height, centred around fixation).

Stimuli were presented on a gamma calibrated CRT display (Dell FP2001, 20.1 inch display; $800 \times 600$ pixels; $60 \mathrm{~Hz}$ refresh rate), at a viewing distance of approximately 60 $\mathrm{cm}$, situated in a darkened room. Stimulus display and response collection were controlled by Matlab 7.8.0 (Mathworks Inc., Natick, MA, USA) using the COGENT 2000 toolbox (http://www.vislab.ucl.ac.uk/cogent.php).

Task

The visual judgement comprised a temporal two-alternative forced choice pop-out task (see Fig. 1 for timings). All the Gabors in one interval were of the same contrast, but in the other interval, one of the Gabors was of a higher contrast than the others (the "popout" Gabor, illustrated by a dashed circle in Fig. 1 that was not present in the actual display). The temporal interval and spatial position of the pop-out Gabor varied randomly between trials. Participants were required to decide whether this pop-out Gabor had appeared in the first or the second interval. The perceptual judgement was indicated by participants using the left hand with the numbers ' 1 ' (first interval) or ' 2 ' (second interval) on the QWERTY keypad of a standard PC keyboard. Participants then indicated their confidence in the perceptual decision they had just made on a scale of 1 (low relative confidence) to 6 (high relative confidence), using their right hand to press 
one of the numbers ' 1 ' to ' 6 ' on the numerical keypad. A square red frame (width 1 degree, thickness 0.1 degree) appeared around the selected rating (Fig. 1).

The contrast of the pop-out Gabor was chosen from the stimulus set of pop-out Gabors using a 1-up 2-down staircase procedure (S1), which at the limit results in convergence on $71 \%$ accuracy. The contrast of the pop-out Gabor at the end of each block was used as the starting contrast for the pop-out Gabor in the next block. Our aim in this staircase procedure was to equate objective perceptual performance across individuals, leaving quantification of metacognitive ability unconfounded by performance (S2).

Participants were instructed to try to use the whole of the confidence scale in their responses, and to bear in mind that the scale represents relative confidence, as, given the difficult nature of the task, they would rarely be completely certain that their visual judgement had been correct. Participants performed a practice session to familiarise themselves with the stimuli and task. The main experiment consisted of 600 trials, split into 6 blocks of 100 trials. They were given no feedback about their performance until the end of the experiment.

\section{Quantification of metacognitive ability}

The accuracy of metacognitive assessments can be intuited as how transparent an initial perceptual decision process is to a putative "higher" level assessment. This intuition can be captured within the logic of signal detection theory (SDT), which assesses how faithfully a creature separates signal from noise. Conventional applications of SDT assess detection performance by comparing the proportion of "hits" and "false alarms" in a stimulus detection task. By applying the logic of SDT to metacognition ("Type 2" SDT), we categorised a "hit" as a high confidence response after a correct decision and a "false alarm" as a high confidence response after an incorrect decision [see table S1 and (S3)]. Because the specific mathematical assumptions of conventional SDT may not hold for this new analysis $(S 4, S 5)$, we used nonparametric assessments of sensitivity and bias (S6). We constructed Type 2 ROC curves for each participant (Fig. 2A and fig. S2) that characterised the probability of being correct for a given level of confidence. ROC curves were anchored at $[0,0]$ and $[1,1]$. An ROC curve that bows sharply upwards indicates that the probability of being correct rises rapidly with confidence; conversely, a flat ROC function indicates a weak link between confidence and accuracy.

We noted a practice effect in the staircase parameters (fig. S1) reflected in a decrease in mean contrast and variability from block 1 to 2. A one-way ANOVA of mean contrast with block as a within-subjects factor revealed a significant effect of block $\left(F_{(5,155)}=8.18, P<0.001\right)$ that was abolished on removal of block $1\left(F_{(4,124)}=\right.$ $1.56, P=0.19)$. ROC analysis was therefore carried out on data from blocks $2-6$, after stabilisation of psychophysical performance. To plot the ROC, $h_{i}=p$ (confidence $=i \mid$ correct) and $f_{i}=p$ (confidence $=i \mid$ incorrect) were calculated for all $i$. These probabilities were then transformed into cumulative probabilities, and plotted against each other (Fig. 2A and fig. S2). Following Kornbrot (S6), we computed distributionfree measures of sensitivity and bias from this ROC by dividing the area into two parts $K_{B}$ is the area between the ROC curve and the major diagonal (solid line in Fig. 2A) to the right of the minor diagonal (dotted line in Fig. 2A), and $K_{A}$ is the area between the 
ROC curve and major diagonal to the left of the minor diagonal. From simple geometry [derived in the Appendix of (S6)], these areas can be calculated as follows:

$$
\begin{aligned}
& K_{A}=\frac{1}{4} \sum_{k=1}^{k=\frac{1}{2} i}\left[\left(h_{k+1}-f_{k}\right)^{2}-\left(h_{k}-f_{k+1}\right)^{2}\right] \\
& K_{B}=\frac{1}{4} \sum_{k=\frac{1}{2} i}^{k=i}\left[\left(h_{k+1}-f_{k}\right)^{2}-\left(h_{k}-f_{k+1}\right)^{2}\right]
\end{aligned}
$$

Sensitivity $\left(A_{\text {roc }}\right)$ is then the sum of these areas, and Type 2 bias $\left(B_{\text {roc }}\right)$ is the log of the ratio:

$$
\begin{gathered}
A_{\text {roc }}=K_{A}+K_{B} \\
B_{\text {roc }}=\ln \left(\frac{K_{A}}{K_{B}}\right)
\end{gathered}
$$

Type I $d^{\prime}$ and bias $(c)$ were calculated in the standard manner (S7):

$d^{\prime}=1 / \sqrt{ } 2[z(H)-z(F)]$

$C=-0.5[z(H)+z(F)]$

where $z$ is the inverse of the cumulative normal distribution function, $H=p$ (response $=$ $1 \mid$ interval $=1)$ and $F=p($ response $=1 \mid$ interval $=2)$. Confirmatory correlation analyses between SDT parameters and grey matter (GM)/fractional anisotropy (FA) clusters [signal extracted using the MarsBar toolbox (S8)] were carried out using Pearson's product-moment correlations in SPSS 17.0.

\section{Voxel-based morphometry analysis}

Voxel-based morphometry (VBM) provides a quantitative measure (at each voxel) of the tissue volume per unit volume of spatially normalised image (S9). A 1.5T Sonata scanner (Siemens Medical Systems, Erlangen, Germany) was used to acquire all images for each participant. T1-weighted anatomical whole-brain scans were acquired for VBM analysis (176 slices, echo time $=3.56 \mathrm{~ms}$, $\mathrm{TR}=12.24 \mathrm{~ms}$, voxel size $1 \mathrm{~mm}$ isotropic). VBM preprocessing was carried out using SPM8 (http://www.fil.ion.ucl.ac.uk/spm). The images were first segmented into GM, white matter (WM) and cerebral spinal fluid in native space (S10). The GM segment images from this process were then rigidly aligned and subsequently warped to an iteratively improved template using nonlinear registration in DARTEL(S11). DARTEL's “Normalise to MNI" module was then used to produce smoothed normalised images. The DARTEL template was affinely registered to MNI space, and the GM images were transformed using the DARTEL flow-fields and this affine transformation, in a way that preserved their local tissue 
volumes (equivalent to a Jacobian "modulation" step). Smoothing used a Gaussian kernel of $8 \mathrm{~mm}$ full width at half maximum.

The pre-processed GM images were entered into a multiple regression model in SPM8 to determine which brain regions showed significant covariation with the SDT-based measures of metacognitive ability. We included $A_{\text {roc }}, d$ ', Type II criterion ( $B_{\text {roc }}$; the overall tendency to use high confidence responses), the absolute (unsigned) value of the Type I criterion $(|c|)$ and gender $(\mathrm{M}=1 ; \mathrm{F}=0)$ in the model. Type I criterion $(c)$ measures the extent of the bias towards interval 1 or 2 on the perceptual decision task, with greater bias reflecting suboptimal performance. Positive values indicate bias towards interval 1, and negative values bias towards interval 2 . We thus entered the absolute value of $c$ as a covariate of no interest, with higher values indicating suboptimal performance bias towards either interval.

Adjustment for "global" brain volume using proportional scaling was applied, resulting in voxel values that were proportions of total GM volume. A binary mask (SPM8 grey.nii template $>0.3$ ) was used to restrict the search volume to changes in GM. Tstatistic maps reflecting the correlation between each regressor and regional GM volume were created. Cluster-based statistics were used to locate significant regions based on both their peak value and spatial extent after applying an initial clusterdefining threshold of $P<0.001$. Due to structural images displaying local variation in smoothness, standard applications of cluster-based random field theory are inappropriate (S12). We thus applied non-stationary cluster extent correction when calculating family-wise error (FWE) corrected $P$ values using the NS toolbox (http://www.fmri.wfubmc.edu/cms/NS-General). Computational simulations (S12) show that for designs with high degrees of freedom and sufficient smoothness, as here, using a cluster defining threshold of $P<0.001$ with correction for non-stationarity provides adequate control over the family-wise false positive rate $(P<0.05)$.

\section{Diffusion tensor imaging analysis}

The diffusion tensor imaging (DTI) dataset comprised of 68 images with 60 slices and $2.3 \mathrm{~mm}$ isotropic resolution. The first 7 images were collected with $\mathrm{b}=100 \mathrm{~s} / \mathrm{mm}^{2}$. The diffusion encoding directions were isotropically distributed on the surface of the sphere (S13) for the remaining 61 images and the b-value was $1000 \mathrm{~s} / \mathrm{mm}^{2}$. The echo time was $90 \mathrm{~ms}$, each 2D image slice took $150 \mathrm{~ms}$ to collect, and the field of view was $220 \mathrm{~mm}$. DTI data sets are often collected using echo-planar imaging (EPI) methods which are affected by susceptibility-induced artefacts. To reduce the extent of these artefacts two datasets were collected for each participant, with the only difference being that the phase encoding direction was reversed for the second run. This method ensures the susceptibility-induced distortions are equal and opposite in the two datasets, providing the opportunity to correct their effect (S14).

Diffusion-weighted images were first aligned using FSL's eddycorrect (http://www.fmrib.ox.ac.uk/fsl/), and then combined into a single dataset with reduced susceptibility-induced artefacts. The main diffusion tensor was then fitted at each voxel using FSL's dtifit. From the tensor a rotationally invariant measure of diffusion anisotropy can be calculated. One such measure is fractional anisotropy (FA) with values ranging from 0 (representing isotropic, or undirected, diffusion) to 1 
(representing a single preferred direction of diffusion). This measure has been used extensively to investigate local WM integrity, as diffusion of water molecules is more restricted perpendicular to, rather than along, neuronal fibres. The calculated FA map for each participant (in native space) was imported into SPM8 and coregistered to the WM segment image of the same participant created during VBM analysis.

Coregistration was carried out by maximising normalised mutual information between the images. The DARTEL flowfields and affine (MNI) transformation were then applied to each participant's coregistered FA image, producing normalised FA images in MNI space. Unlike the VBM normalisation (which preserved the original local tissue volume), the FA images were normalised in a way that preserved their original voxel values (without "modulation"). Normalised FA images were also smoothed with a $8 \mathrm{~mm}$ full-width at half maximum Gaussian kernel prior to statistical analysis. For one participant, DTI scans were unavailable, leaving 30 subjects in the FA analysis.

Statistical analysis of FA proceeded in an identical fashion to that of GM volume (see above). A multiple regression model was constructed consisting of $A_{\text {roc }}, d^{\prime}$, Type II criterion ( $B_{\text {roc }}$; the overall tendency to use high confidence responses) and the absolute (unsigned) value of the Type I criterion $(|c|)$. A binary mask (mean normalised FA $>$ 0.2 ) was used to restrict the search volume to changes in WM. Statistical inference was conducted as for VBM. Probable tract labels were obtained using the JHU White-Matter Tractography Atlas within FSL.

\section{ROC model fits}

To explore how well a Gaussian Type II SDT model accounted for the confidence rating data (S7), we fit the following linear regression model:

$z(h)=\beta_{0}+\beta_{1} z(f)+\varepsilon$

where $z$ is the inverse of the cumulative normal distribution function. This model provided an excellent fit to the data (mean $R^{2}=0.97$ ), indicating that the underlying $f(X \mid$ correct $)$ and $f(X \mid$ incorrect $)$ distributions are normal-like [where $X$ is a random decision variable; see (S4) for further details]. The $\beta_{1}$ parameter (slope) indicates the relative variance of the two distributions. This parameter was on average less than 1 within our sample $(0.88 \pm 0.026 \mathrm{SEM})$, indicating that the $f(X \mid$ correct $)$ distribution has greater variance than the $f(X \mid$ incorrect $)$. Interestingly, theoretical models that suggest a direct translation of Type I into Type II distributions predict a Type II ROC slope slightly less than 1 (S4). However, this picture is not clear-cut: recent metamemory data support an equal-variance Gaussian model(S15). We note that our use of nonparametric methods to characterise $A_{\text {roc }}$ are not dependent on the specific form of the model used; indeed, it was the methodological uncertainty surrounding the quantification of Type II processes that led us to adopt the distribution-free approach (S6).

\section{Control analyses of GM and FA correlations}

We carried out additional analysis to rule out potential alternative interpretations of our findings. One concern is that variation in underlying perceptual acuity could confound the anatomical variance we ascribe to metacognitive ability $\left(A_{\text {roc }}\right)$. Good perceptual ability may be reflected in low mean stimulus contrast and/or low staircase variability 
(though we note that extraneous environmental or ocular factors also affect these variables). To rule out this interpretation, we computed the partial correlation between brain structure and $A_{\text {roc }}$ while controlling for both mean stimulus contrast and the variability (SD) in the staircase required to achieve a constant level of performance within each individual. Both the GM cluster in BA10 $(r=0.39, P=0.036)$ and the FA cluster in anterior corpus callosum $(r=0.74, P<0.001)$ remained significantly correlated with $A_{\text {roc }}$ after controlling for mean contrast and staircase variability.

This partial correlation analysis only examines the correlation of predefined regions. As a further test, we constructed a second design matrix in which mean stimulus contrast and staircase variability were directly entered as predictors of GM/FA, with gender again present as a covariate of no interest. Neither measure correlated with grey matter or FA at the statistical thresholds used in the main analysis $(P>0.05$, corrected for multiple comparisons), even when applying a mask ( $8 \mathrm{~mm}$ sphere) to isolate voxels within the vicinity of the BA10 (GM) or the anterior corpus callosum (FA) peak voxels. While we are cautious in interpreting uncorrected findings, one result of potential interest is that GM volume in the medial calcarine sulcus, consistent with the location of early visual cortex, showed increased volume in subjects with greater perceptual acuity as defined by negative mean stimulus contrast $(P<0.001$, uncorrected). Table S4 details uncorrected results from these models for completeness. Together these control analyses indicate that the correlations we observe between $A_{r o c}$ and structure relate to differences in metacognitive ability rather than low-level differences in performance.

\section{Negative correlations with $A_{\text {roc }}$}

We found negative correlations with $A_{r o c}$ in bilateral regions of anterior inferior temporal grey matter (left, $P<0.05$, corrected for multiple comparisons; right, $P<$ 0.001 , uncorrected; table S3). While we are cautious about interpreting the relevance of a decrease in grey matter volume for increased metacognitive ability, we note that these temporopolar regions have been implicated in both self-related(S16) and higher-order visual (S17) processing, and thus alterations in grey matter here might similarly place functional constraints on perceptual metacognition. 


\section{Figure S1}
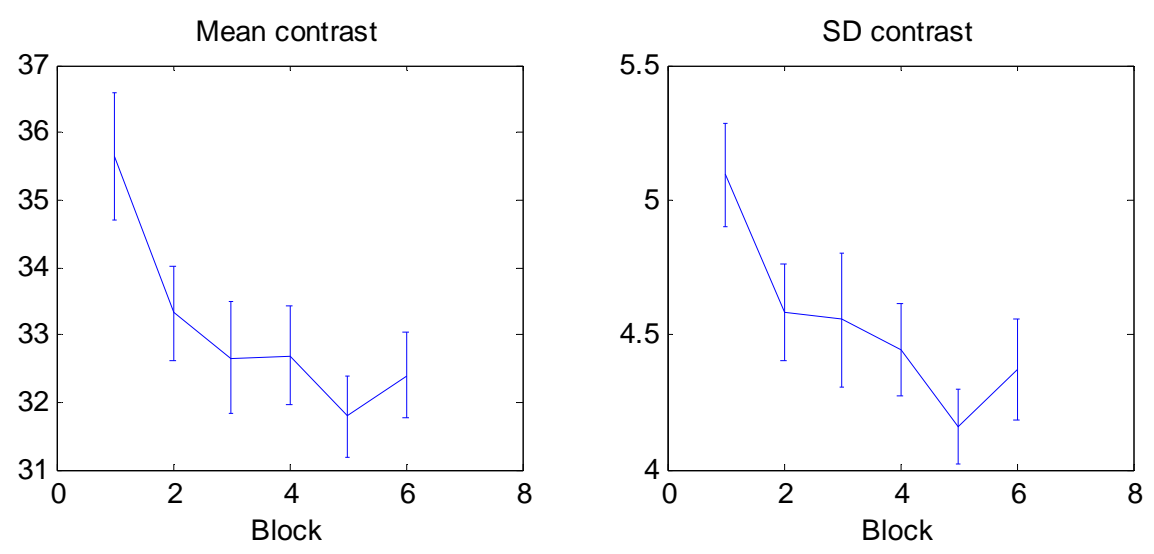

Stimulus parameters for the behavioural task. Mean and standard deviation (SD) of oddball Gabor contrast (percentage of maximum contrast) plotted for each block of the perceptual task, averaged over participants. Error bars represent one standard error of the mean. Because stimulus contrast and variability were significantly higher in Block 1 (see Methods), indicating a period of gradual stabilisation of performance, only data from blocks 2-6 were used to calculate SDT measures. 


\section{Figure S2}
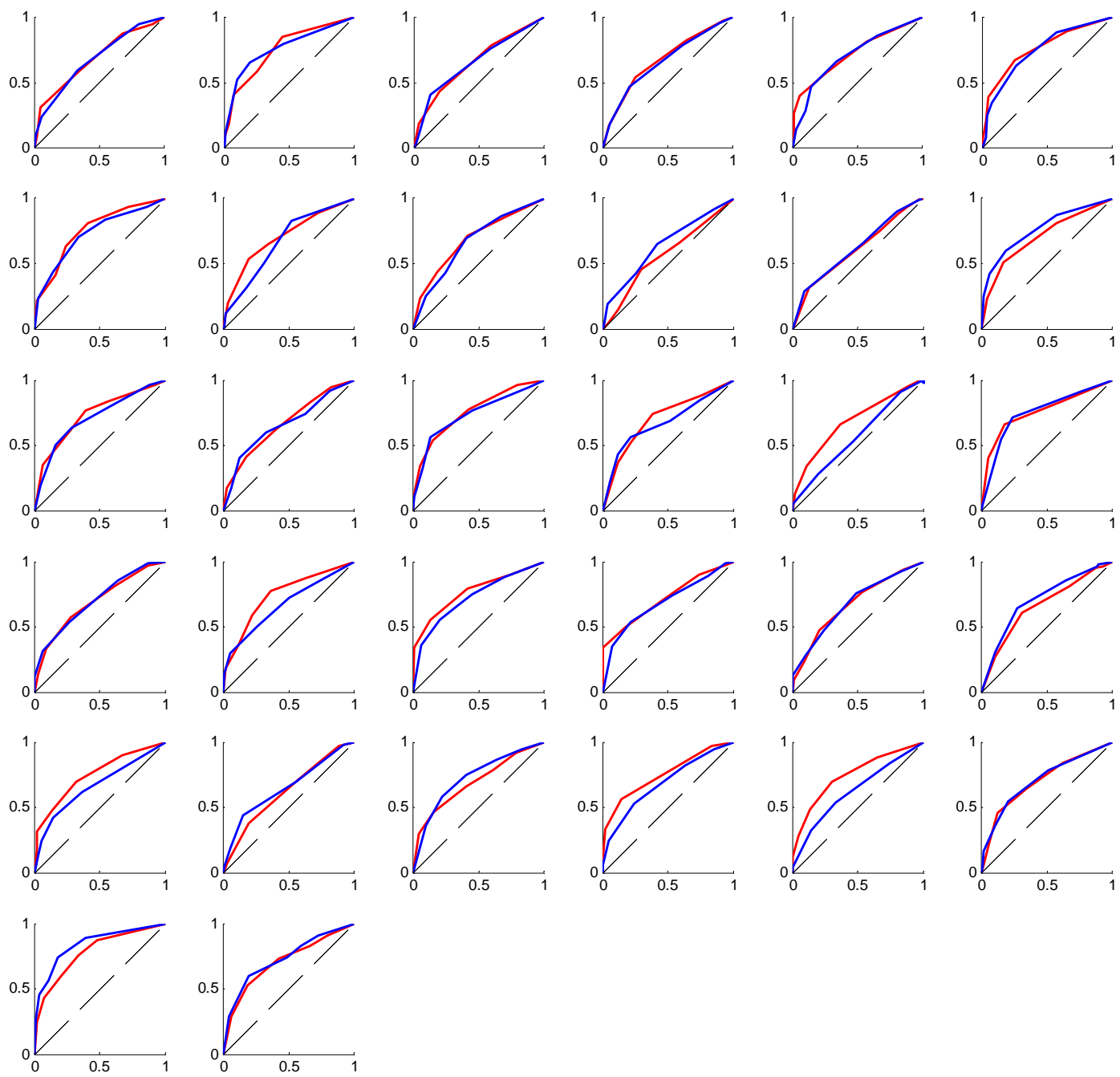

Individual ROC curves calculated from the behavioural data (see Materials and Methods \& Fig. 2) plotted for each of the 32 participants, split into odd (blue; blocks 3 and 5) and even (red; blocks 2, 4 and 6) blocks of the psychophysics session. 


\section{Figure S3}

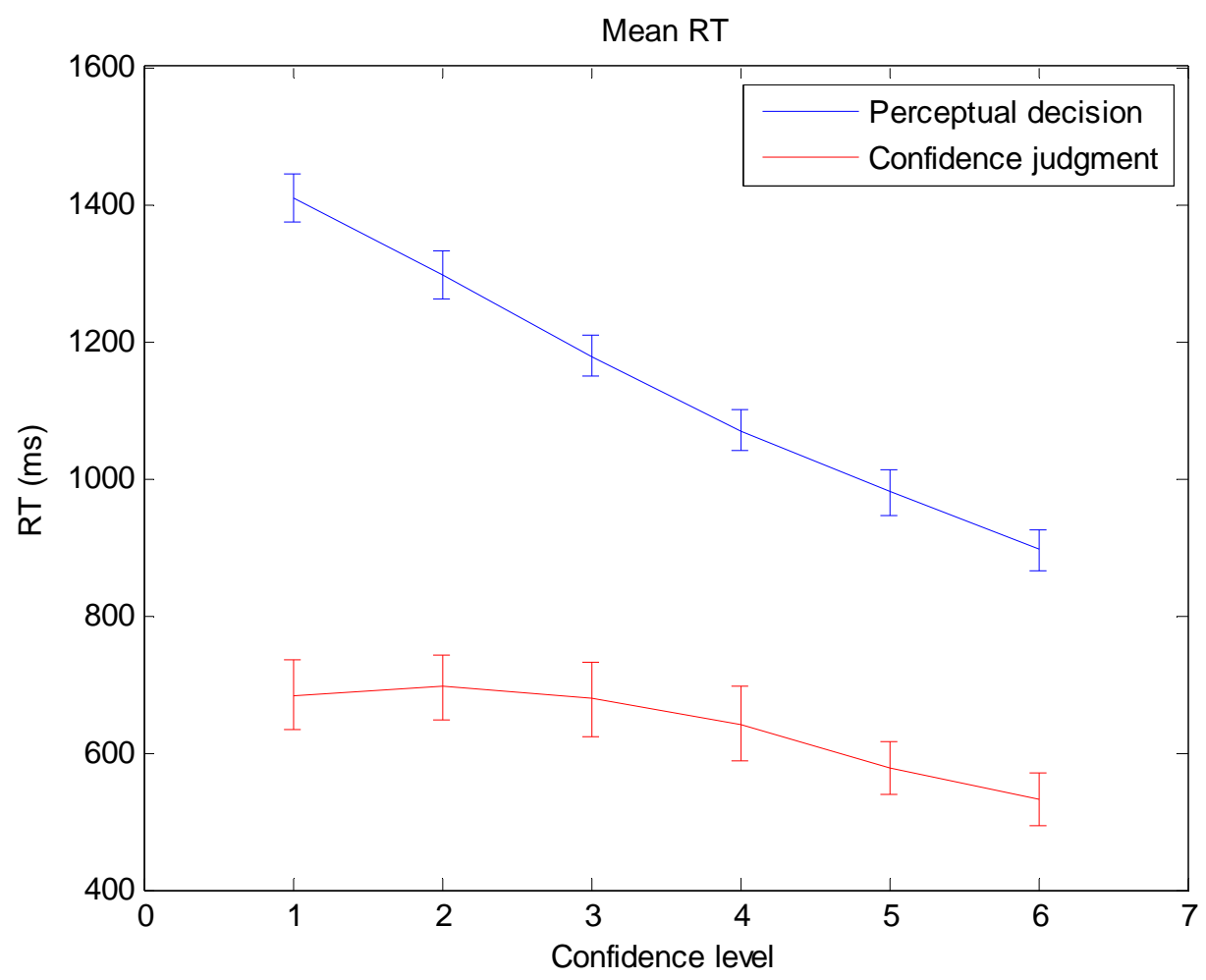

Mean reaction times (RT) measured in milliseconds for both the perceptual decision (blue) and the confidence judgment (red) from blocks 2-6, plotted as a function of reported confidence level. Data are averaged across 32 participants and the error bars represent one standard error of the mean. 


\section{Figure S4}

(A)

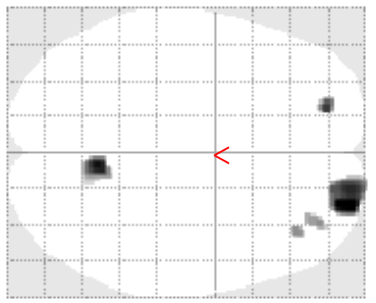

(B)

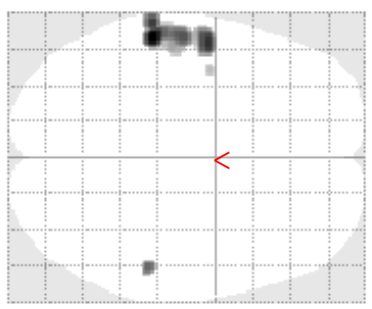

(C)

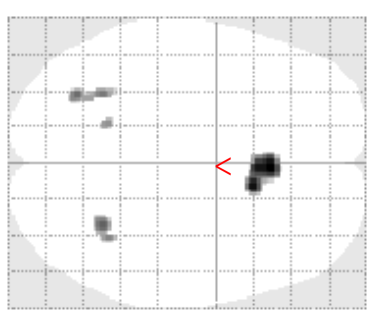

Axial "glass brains" (viewed from above) showing areas where grey matter volume correlates positively (A) and negatively (B) with $A_{r o c}$, and where white matter fractional anisotropy correlates positively with $A_{\text {roc }}$ (C). No suprathreshold fractional anisotropy clusters were found for negative correlations with $A_{\text {roc }}$ (see also tables S2 and S3). All maps are thresholded at $P<0.001$, uncorrected with an extent threshold of 10 voxels. 
Table S1

Classification of responses within Type II signal detection theory, assuming binary confidence ratings. In our task, we used graded confidence ratings, allowing computation of Type II sensitivity from the full ROC function.

\begin{tabular}{c||c|c} 
Type I decision & High confidence & Low confidence \\
\hline \hline Correct & Hit & Miss \\
\hline Incorrect & False alarm & Correct rejection
\end{tabular}




\section{Table S2}

GM volume associated with behavioural variables (SDT parameters) entered into the multiple regression model. Whole-brain corrected clusters $(P<0.05$, corrected for multiple comparisons) are indicated in bold type. For completeness, correlations that survive a height threshold of $P<0.001$, uncorrected, and an extent threshold of 10 voxels are also reported. Abbreviations: $\mathrm{PFC}$ - prefrontal cortex; BA - Brodmann area.

\begin{tabular}{|c|c|c|c|c|c|c|}
\hline Regressor & $\begin{array}{c}\text { Number } \\
\text { of } \\
\text { voxels }\end{array}$ & $\begin{array}{c}\text { Peak } \\
\text { voxel } \\
\text { Z- } \\
\text { score } \\
\end{array}$ & $\begin{array}{c}P \text { value } \\
\text { (cluster } \\
\text { FWE } \\
\text { corrected) } \\
\end{array}$ & $\begin{array}{l}\text { Peak voxel } \\
\text { MNI } \\
\text { coordinates }\end{array}$ & Laterality & Label \\
\hline \multirow{5}{*}{$A_{\text {roc }}$} & 675 & 4.02 & 0.029 & 246518 & $\mathbf{R}$ & \multirow{5}{*}{$\begin{array}{c}\text { Anterior PFC (BA10) } \\
\text { Precuneus/posterior } \\
\text { cingulate } \\
\text { Anterior PFC (BA10) } \\
\text { Dorsolateral PFC } \\
\text { (BA46) } \\
\text { Anterior PFC (BA10) }\end{array}$} \\
\hline & 291 & 3.93 & 0.191 & $6-5718$ & $\mathrm{~L} / \mathrm{R}$ & \\
\hline & 31 & 3.78 & 0.703 & -205312 & $\mathrm{~L}$ & \\
\hline & 25 & 3.45 & 0.829 & 363921 & $\mathrm{R}$ & \\
\hline & 29 & 3.44 & 0.497 & 33509 & $\mathrm{R}$ & \\
\hline \multirow{4}{*}{$\begin{array}{l}\text { Negative } \\
\quad A_{\text {roc }}\end{array}$} & 713 & 3.92 & 0.026 & $-56-30-26$ & $\mathbf{L}$ & \multirow{4}{*}{$\begin{array}{c}\begin{array}{c}\text { Inferior temporal } \\
\text { gyrus }\end{array} \\
\text { Superior Temporal } \\
\text { gyrus } \\
\text { Inferior temporal gyrus } \\
\text { Inferior temporal gyrus }\end{array}$} \\
\hline & 76 & 3.69 & 0.753 & $-63-3010$ & $\mathrm{~L}$ & \\
\hline & 80 & 3.54 & 0.457 & $51-33-21$ & $\mathrm{R}$ & \\
\hline & 15 & 3.22 & 0.995 & $-41-3-48$ & $\mathrm{~L}$ & \\
\hline$B_{\text {roc }}$ & 28 & 3.93 & 0.313 & $-33-7334$ & $\mathrm{~L}$ & Occipital lobe (BA19) \\
\hline \multirow{2}{*}{$\begin{array}{l}\text { Negative } \\
B_{\text {roc }}\end{array}$} & 93 & 3.47 & 0.233 & $-59-27-14$ & $\mathrm{~L}$ & \multirow{2}{*}{$\begin{array}{c}\text { Middle temporal gyrus } \\
\text { Superior temporal } \\
\text { gyrus }\end{array}$} \\
\hline & 20 & 3.35 & 0.826 & $-66-103$ & $\mathrm{~L}$ & \\
\hline \multirow{5}{*}{$d^{\prime}$} & 82 & 3.77 & 0.175 & $-3-84-21$ & $\mathrm{~L} / \mathrm{R}$ & \multirow{5}{*}{$\begin{array}{c}\text { Cerebellum } \\
\text { Middle temporal sulcus } \\
\text { Superior parietal } \\
\text { Lingual gyrus } \\
\text { Supplementary motor } \\
\text { area (BA6) }\end{array}$} \\
\hline & 16 & 3.68 & 0.939 & $53-25-15$ & $\mathrm{R}$ & \\
\hline & 389 & 3.66 & 0.112 & $60-3951$ & $\mathrm{R}$ & \\
\hline & 47 & 3.45 & 0.817 & $6-614$ & $\mathrm{~L} / \mathrm{R}$ & \\
\hline & 18 & 3.26 & 0.953 & $-3-966$ & $\mathrm{~L}$ & \\
\hline $\begin{array}{c}\text { Negative } \\
\text { d' }\end{array}$ & N/A & N/A & $\mathrm{N} / \mathrm{A}$ & N/A & N/A & $\begin{array}{c}\text { No suprathreshold } \\
\text { clusters }\end{array}$ \\
\hline
\end{tabular}




\section{Table S3}

White matter microstructure (fractional anisotropy; FA) associated with behavioural variables (SDT measures) entered into the multiple regression model. Clusters that survive correction for multiple comparisons $(P<0.05)$ are indicated in bold type. For completeness, correlations that survive a height threshold of $P<0.001$, uncorrected, and an extent threshold of 10 voxels are also reported.

\begin{tabular}{|c|c|c|c|c|c|c|}
\hline Regressor & $\begin{array}{c}\text { Number } \\
\text { of } \\
\text { voxels }\end{array}$ & $\begin{array}{c}\text { Peak } \\
\text { voxel } \\
\text { Z- } \\
\text { score }\end{array}$ & $\begin{array}{c}P \text { value } \\
\text { (cluster } \\
\text { FWE } \\
\text { corrected) }\end{array}$ & $\begin{array}{l}\text { Peak voxel } \\
\text { MNI } \\
\text { coordinates }\end{array}$ & Laterality & Label \\
\hline \multirow{6}{*}{$A_{\text {roc }}$} & 308 & 3.93 & 0.033 & $226-2$ & $\mathbf{L} / \mathbf{R}$ & Genual corpus callosum \\
\hline & 66 & 3.58 & 0.492 & $29-55-2$ & $\mathrm{R}$ & $\begin{array}{l}\text { Posterior corpus callosum } \\
\text { (forceps major) }\end{array}$ \\
\hline & 31 & 3.54 & 0.502 & $-32-670$ & $\mathrm{~L}$ & $\begin{array}{l}\text { Inferior fronto-occipital } \\
\text { fasciculus }\end{array}$ \\
\hline & 26 & 3.48 & 0.680 & $-32-5514$ & $\mathrm{~L}$ & Longitudinal fasciculus \\
\hline & 13 & 3.44 & 0.824 & $35-52-15$ & $\mathrm{R}$ & $\begin{array}{l}\text { Inferior longitudinal } \\
\text { fasciculus }\end{array}$ \\
\hline & 11 & 3.39 & 0.631 & $-18-5228$ & $\mathrm{~L}$ & Cingulum \\
\hline $\begin{array}{c}\text { Negative } \\
A_{\text {roc }}\end{array}$ & $\mathrm{N} / \mathrm{A}$ & $\mathrm{N} / \mathrm{A}$ & $\mathrm{N} / \mathrm{A}$ & $\mathrm{N} / \mathrm{A}$ & N/A & No suprathreshold clusters \\
\hline$B_{\text {roc }}$ & $\mathrm{N} / \mathrm{A}$ & $\mathrm{N} / \mathrm{A}$ & $\mathrm{N} / \mathrm{A}$ & $\mathrm{N} / \mathrm{A}$ & $\mathrm{N} / \mathrm{A}$ & No suprathreshold clusters \\
\hline \multirow{3}{*}{$\begin{array}{c}\text { Negative } \\
B_{\text {roc }}\end{array}$} & 128 & 4.24 & 0.226 & $-820-9$ & $\mathrm{~L}$ & Genual corpus callosum \\
\hline & 49 & 3.91 & 0.132 & $26-51-9$ & $\mathrm{R}$ & Posterior corona radiata \\
\hline & 21 & 3.54 & 0.438 & -182924 & $\mathrm{~L}$ & Cingulum \\
\hline \multirow{2}{*}{$d^{\prime}$} & 74 & 3.89 & 0.251 & -17639 & $\mathrm{~L}$ & Superior corona radiata \\
\hline & 18 & 3.33 & 0.721 & $-18-745$ & $\mathrm{~L}$ & Superior corona radiata \\
\hline $\begin{array}{l}\text { Negative } \\
d^{\prime}\end{array}$ & N/A & $\mathrm{N} / \mathrm{A}$ & $\mathrm{N} / \mathrm{A}$ & $\mathrm{N} / \mathrm{A}$ & $\mathrm{N} / \mathrm{A}$ & No suprathreshold clusters \\
\hline
\end{tabular}




\section{Table S4}

GM correlating with negative stimulus contrast and staircase variability (low-level measures of perceptual performance). After correcting for multiple comparisons, no significant clusters were observed, but correlations that survive a height threshold $P<$ 0.001 , uncorrected, and an extent threshold of 10 voxels are reported for completeness.

\begin{tabular}{|c|c|c|c|c|c|c|c|}
\hline Analysis & Regressor & $\begin{array}{c}\text { Number } \\
\text { of } \\
\text { voxels }\end{array}$ & $\begin{array}{l}\text { Peak } \\
\text { voxel } \\
\text { Z- } \\
\text { score }\end{array}$ & $\begin{array}{c}\text { P value } \\
\text { (cluster } \\
\text { FWE } \\
\text { corrected) }\end{array}$ & $\begin{array}{c}\text { Peak voxel } \\
\text { MNI } \\
\text { coordinates }\end{array}$ & Laterality & Label \\
\hline \multirow{9}{*}{ GM } & \multirow{5}{*}{$\begin{array}{l}\text { Negative } \\
\text { mean } \\
\text { contrast }\end{array}$} & 88 & 3.67 & 0.917 & $14-1024$ & $\mathrm{R}$ & Caudate \\
\hline & & 51 & 3.56 & 0.783 & $-65-574$ & $\mathrm{~L}$ & $\begin{array}{l}\text { Middle } \\
\text { temporal gyrus }\end{array}$ \\
\hline & & 78 & 3.44 & 0.913 & $5-7621$ & $\mathrm{~L} / \mathrm{R}$ & $\begin{array}{c}\text { Calcarine } \\
\text { sulcus }\end{array}$ \\
\hline & & 80 & 3.38 & 0.908 & 33642 & $\mathrm{~L} / \mathrm{R}$ & $\begin{array}{l}\text { Dorsal medial } \\
\text { prefrontal } \\
\text { cortex }\end{array}$ \\
\hline & & 11 & 3.21 & 0.982 & $-1429-20$ & $\mathrm{~L}$ & $\begin{array}{c}\text { Orbitofrontal } \\
\text { cortex }\end{array}$ \\
\hline & \multirow{4}{*}{$\begin{array}{l}\text { Negative } \\
\text { SD }\end{array}$} & 128 & 4.00 & 0.301 & $59-421$ & $\mathrm{R}$ & $\begin{array}{c}\text { Middle } \\
\text { temporal gyrus }\end{array}$ \\
\hline & & 29 & 3.70 & 0.577 & $-51-3336$ & $\mathrm{~L}$ & $\begin{array}{l}\text { Inferior } \\
\text { parietal }\end{array}$ \\
\hline & & 34 & 3.36 & 0.938 & $47-15-48$ & $\mathrm{R}$ & $\begin{array}{l}\text { Postcentral } \\
\text { gyrus }\end{array}$ \\
\hline & & 22 & 3.24 & 0.953 & $-44-2146$ & $\mathrm{~L}$ & $\begin{array}{c}\begin{array}{c}\text { Postcentral } \\
\text { gyrus }\end{array} \\
\end{array}$ \\
\hline \multirow[t]{2}{*}{ FA } & $\begin{array}{c}\text { Negative } \\
\text { mean } \\
\text { contrast } \\
\end{array}$ & N/A & N/A & N/A & N/A & N/A & $\begin{array}{c}\text { No } \\
\begin{array}{c}\text { suprathreshold } \\
\text { clusters }\end{array} \\
\end{array}$ \\
\hline & $\begin{array}{l}\text { Negative } \\
\text { SD }\end{array}$ & 30 & 3.86 & 0.942 & $-29-1548$ & $\mathrm{~L}$ & $\begin{array}{c}\text { Superior } \\
\text { corona radiata }\end{array}$ \\
\hline
\end{tabular}




\section{References}

S1. H. Levitt, Transformed up-down methods in psychoacoustics. J. Acoust. Soc. Am. 49, Suppl (1971).

S2. H.C. Lau, in Frontiers of Consciousness, Eds. L. Weiskrantz, M. Davies (Oxford University Press: 2008), pp. 245-258.

S3. C. Kunimoto, J. Miller, H. Pashler, Confidence and accuracy of near-threshold discrimination responses. Conscious. Cogn. 10, 294 (2001).

S4. S.J. Galvin, J.V. Podd, V. Drga, J. Whitmore, Type 2 tasks in the theory of signal detectability: discrimination between correct and incorrect decisions. Psychon. Bull. Rev. 10, 843 (2003).

S5. S. Evans, P. Azzopardi, Evaluation of a 'bias-free' measure of awareness. Spat.Vis. 20,61 (2007).

S6. D.E. Kornbrot, Signal detection theory, the approach of choice: model-based and distribution-free measures and evaluation. Percept. Psychophys. 68, 393 (2006).

S7. N. Macmillan, C. Creelman, Detection theory: a user's guide. (Lawrence Erlbaum: New York, 2005).

S8. M. Brett, J. Anton, R. Valabregue, J. Poline, Regions of interest analysis using an SPM toolbox. Presented at the 8th International Conference on Functional Mapping of the Human Brain (2002).

S9. J. Ashburner, K.J. Friston, Voxel-based morphometry--the methods. NeuroImage 11, 805 (2000).

S10. J. Ashburner, K.J. Friston, Unified segmentation. NeuroImage 26, 839 (2005).

S11. J. Ashburner, A fast diffeomorphic image registration algorithm. NeuroImage 38, 95 (2007).

S12. S. Hayasaka, K.L. Phan, I. Liberzon, K.J. Worsley, T.E. Nichols, Nonstationary cluster-size inference with random field and permutation methods. NeuroImage 22, 676 (2004).

S13. K.M. Jansons, D.C. Alexander, Persistent angular structure: new insights from diffusion MRI data. Inf. Process. Med. Imaging 18, 672 (2003).

S14. J.L.R. Andersson, S. Skare, J. Ashburner, How to correct susceptibility distortions in spin-echo echo-planar images: application to diffusion tensor imaging. NeuroImage 20, 870 (2003).

S15. P.A. Higham, No special K! A signal detection framework for the strategic 
regulation of memory accuracy. J. Exp. Psychol. Gen. 136, 1 (2007).

S16. U. Frith, C.D. Frith, Development and neurophysiology of mentalizing. Philos. T. R. Soc. B. 358, 459 (2003).

S17. C.G. Gross, S.D. Schonen, Representation of visual stimuli in inferior temporal cortex. Philos. T. R. Soc. B. 335, 3 (1992). 